\title{
Vitreoretinal traction is a major factor in causing the haemorrhagic retinopathy of abusive head Injury? - Yes
}

Eye (2009) 23, 1758-1760; doi:10.1038/eye.2009.199; published online 7 August 2009

Retinal haemorrhage is a cardinal manifestation of abusive head injury characterized by repetitive acceleration-deceleration forces with or without blunt head impact (Shaken Baby syndrome). That shaking alone, in the absence of head impact, can result in severe, if not fatal, manifestations of this syndrome has been well demonstrated. ${ }^{1,2}$ The unique nature of repetitive accelerationdeceleration forces in accumulating forces experienced by the eyes has been supported by finite element analysis and corresponds to the areas of the eye where there is a predilection for retinal haemorrhage to appear in abusive injury: the peripheral and macular retina. ${ }^{3,4}$ Isolated accidental blunt head impact injury is rarely associated with retinal haemorrhage, $e^{5,6}$ and when retinal haemorrhage does occur, the haemorrhages are few in number and largely confined to the posterior pole, with the exception perhaps of fatal head crush injury ${ }^{7,8}$ and severe fatal motor vehicle accidents. ${ }^{9}$

Over the last 35 years much research has been conducted to aid us in understanding the pathophysiology of retinal haemorrhage in abusive head injury. Multiple research lenses have been used to examine this issue and each continues to indicate the important role of vitreoretinal traction. In part, this realization comes from the absence of viable alternative theory. There appears to be no correlation between retinal haemorrhage in abusive head injury and intracranial haemorrhage, increased intracranial pressure, or increased intrathoracic pressure. ${ }^{10}$ Although intracranial haemorrhage is commonly associated with retinal haemorrhage (Terson's syndrome) in adults, it appears to be rare in childhood ${ }^{11}$ and not associated with the severe haemorrhagic retinopathy seen in approximately two-thirds of abusive head injury characterized by repetitive acceleration-deceleration forces. ${ }^{10}$ Theories of increased intracranial or intrathoracic pressure leading to a restriction of venous outflow from the eye do not explain the absence of a clinical pattern of retinal haemorrhage consistent with venous obstruction in abused children and the very low incidence of papilledema. ${ }^{10,12}$ Models of increased intrathoracic pressure, which would theoretically result in retinal haemorrhage through a restriction of venous outflow, if not retrograde flow, such as coughing, ${ }^{13}$ vomiting, ${ }^{14}$ and seizures ${ }^{15}$ also fail to demonstrate retinal haemorrhage.

The distribution of retinal haemorrhage in abusive head injury parallels the vitreoretinal anatomy of infants, whereby the greatest adhesive forces are found in the peripheral and macular retina. These adhesive forces are particularly strong in infants. It was perhaps Greenwald et al $^{16}$ who first recognized the importance of vitreoretinal traction when they described traumatic retinoschisis, a physical splitting of the retinal layers from this tension. Multiple studies have recognized the importance of this lesion, which may be accompanied by surrounding folds in the retina, which on postmortem specimens may still have vitreous attached at their apices. In a cat model, vitreoretinal shear has been shown to disrupt retinal vascular autoregulation, leading to patulous vessels that would be more likely to bleed. ${ }^{17}$ In humans, the unique damage to orbital tissues in settings of abusive repetitive acceleration-deceleration forces may also contribute to autonomic vascular dysregulation. ${ }^{18}$ 
Unfortunately, some researchers have tried to generate hypotheses based on impure data. A typical error is the mixing of various types of abuse to draw conclusions about the specific form of abusive head injury in which repetitive acceleration-deceleration forces have the major function. One large study examined 43 years of pathologic specimens, dating well before Shaken Baby syndrome was even recognized and including a multitude of fatal abusive injuries ranging up to 9 years old (an age well beyond the usual range for Shaken Baby syndrome), to suggest theories of retinal haemorrhage causation other than vitreoretinal traction. ${ }^{19}$ The researchers found retinal haemorrhage in only $46 \%$ of children at autopsy, far below the near $100 \%$ found in most postmortem studies, and a high incidence of papilledema, both confirming that indeed they were looking at a heterogeneous sample that included abusive injury from other causes such as pure blunt impact. Yet when one tries to dissect their results to isolate those children who were victims of the specific form of abusive head injury that interests us here, once again the findings of injury that characterizes the results of repetitive acceleration-deceleration forces and vitreoretinal traction-induced injury appear vitreous attached to retinoschisis lesions, intrascleral haemorrhage at the optic nerve-sclera junction, optic atrophy that can be unilateral, and six of the seven children with confessed 'shaking' who had severe haemorrhagic retinopathy.

Research examining the ocular manifestations of abusive head injury using a variety of strategies, including analysis of affected children both pre- and postmortem, examination of nonaffected children with clinical conditions, which in some way recapitulate aspects of the syndrome, animal models, mechanical models, and computer models, has independently directed us to the understanding of the critical role of vitreoretinal traction in this severe and unique form of injury resulting in retinal haemorrhage. The forces that perpetrators use on these children are far beyond that which occurs in the normal (and even surgical) 'life of an eyeball' and lead to a characteristic pattern of haemorrhagic retinopathy observed only very rarely in this age group from other causes. Perhaps the only frequent situation in which such severity of retinal haemorrhage can be observed, interestingly always in the absence of retinoschisis, is normal birth, in which the pathogenesis of the haemorrhage has been ascribed to the effect of prostaglandins, ${ }^{20}$ which have a function in vascular autoregulation, thus providing a possible link to the biochemical pathway of retinal haemorrhage generation in abuse. The roles of other factors, such as increased intracranial pressure, increased intrathoracic pressure, hypoxia, serum sodium imbalance, thrombophilia, and coagulopathy, seem to be secondary, if at all involved, but may be important in perhaps modulating the degree of retinal haemorrhage in any given child.

\section{Conflict of interest}

The authors declare no conflict of interest.

\section{References}

1 Alexander R, Sato Y, Smith W, Bennett T. Incidence of impact trauma with cranial injuries ascribed to shaking. Am J Dis Child 1990; 144: 724-726.

2 Gill JR, Goldfeder LB, Armbrustmacher V, Coleman A, Mena $\mathrm{H}$, Hirsch CS. Fatal head injury in children younger than 2 years in New York City and an overview of the Shaken Baby syndrome. Arch Pathol Lab Med 2009; 133: 619-627.

3 Rangarajan N, Kamalakkannan S, Hasija H, Shams T, Jenny C, Serbanescu I et al. Finite element model of ocular injury in Shaken Baby syndrome. J AAPOS 2009 (in press).

4 Bhola R, Cirovic S, Parson M, Hose D, Lawford P, Howard I. Modeling of the eye and orbit to simulate Shaken Baby syndrome. Invest Ophthalmol Vis Sci 2005; 46: e-abstract 4090.

5 Bechtel K, Stoessel K, Leventhal JM, Ogle E, Teague B, Lavietes $\mathrm{S}$ et al. Characteristics that distinguish accidental from abusive injury in hospitalized young children with head trauma. Pediatrics 2004; 114: 165-168.

6 Levin A. Retinal haemorrhage and child abuse. In: David T (ed). Recent Advances in Paediatrics. Churchill Livingstone: London, 2000, pp 151-219.

7 Lantz PE, Sinal SH, Stanton CA, Weaver Jr RG. Perimacular retinal folds from childhood head trauma. $\mathrm{Br}$ Med J 2004; 328: 754-756.

8 Lueder GT, Turner JW, Paschall R. Perimacular retinal folds simulating nonaccidental injury in an infant. Arch Ophthalmol 2006; 124: 1782-1783.

9 Kivlin JD, Currie ML, Greenbaum VJ, Simons KB, Jentzen J. Retinal hemorrhages in children following fatal motor vehicle crashes: a case series. Arch Ophthalmol 2008; 126: 800-804.

10 Morad Y, Kim Y, Armstrong D, Huyer D, Mian M, Levin A. Correlation between retinal abnormalities and intracranial abnormalities in the Shaken Baby syndrome. Am J Ophthalmol 2002; 134: 354-359.

11 Schloff S, Mullaney P, Armstrong D, Simantirakis E, Humphreys RP, Myseros JS et al. Retinal findings in children with intracranial hemorrhage. Ophthalmology 2002; 109: 1472-1476.

12 Kivlin J, Simons K, Lazoritz S, Ruttum M. Shaken Baby syndrome. Ophthalmology 2000; 107: 1246-1254.

13 Goldman M, Dagan Z, Yair M, Elbaz U, Lahat E, Yair M. Severe cough and retinal hemorrhage in infants and young children. J Pediatr 2006; 148: 835-836.

14 Herr S, Pierce M, Berger R, Ford H, Pitetti R. Does Valsalva retinopathy occur in infants? An initial investigation in infants with vomiting caused by pyloric stenosis. Pediatrics 2004; 113: 1658-1661.

15 Tyagi A, Scotcher S, Kozeis N, Willshaw H. Can convulsions alone cause retinal haemorrhages in infants? Br J Ophthalmol 1998; 82: 659-660. 
16 Greenwald M, Weiss A, Oesterle C, Friendly D. Traumatic retinoschisis in battered babies. Ophthalmology 1986; 93 : 618-625.

17 Nagaoka T, Sakamoto T, Mori F, Sato E, Yoshida A. The effect of nitric oxide on retinal blood flow during hypoxia in cats. Invest Ophthalmol Vis Sci 2002; 43: 3037-3044.

18 Wygnanski-Jaffe T, Levin AV, Shafiq A, Smith C, Enzenauer RW, Elder JE et al. Postmortem orbital findings in Shaken Baby syndrome. Am J Ophthalmol 2006; 142: 233-240 e2.

19 Emerson MV, Jakobs E, Green WR. Ocular autopsy and histopathologic features of child abuse. Ophthalmology 2007; 114: 1384-1394.

20 Gonzalez Viejo I, Ferrer Novella C, Pueyo Subias M, Ronchera Oms JM, Bueno Lozano J, Ferrer Novella E et al. Hemorrhagic retinopathy in newborns: frequency, form of presentation, associated factors and significance. Eur J Ophthalmol 1995; 5: 247-250.

\author{
Pediatric Ophthalmology and Ocular Genetics, \\ Wills Eye Institute, Thomas \\ Jefferson University, \\ Philadelphia, PA, USA \\ Correspondence: AV Levin, \\ Pediatric Ophthalmology and Ocular Genetics, \\ Wills Eye Institute, \\ Suite 1210, 840 Walnut Street, \\ Philadelphia, PA 19107-5109, USA \\ Tel: + 215928 3914; \\ Fax: + 2159283983. \\ E-mail: alevin@willseye.org
}

